\title{
Codificações Espaço-Temporais Tensoriais em Sistemas MIMO Sem Fio
}

\author{
Bárbara da Silva Oliveira, Michel Gonzaga dos Santos e Walter da Cruz Freitas Júnior
}

Resumo-Este artigo de iniciação científica tem como objetivo o estudo da aplicação de técnicas de codificação espaço-temporal baseados em produtos de Khatri-Rao e de Kronecker em sistemas MIMO. O desempenho desses modelos é comparado ao de outros algoritmos clássicos.

\section{Keywords-MIMO, Khatri-Rao,Kronecker}

Abstract-This undergraduate paper aims to to study the application of space-time coding techniques based on Khatri-Rao and Kronecker products in MIMO systems. The performance of these models are compared to other classic algorithms.

\section{Keywords-MIMO, Khatri-Rao,Kronecker}

\section{INTRODUÇÃO}

Sistemas de comunicação sem fio de múltiplas entradas e múltiplas saídas (MIMO) proporcionam maior robustez e altas taxas de transmissão de dados, características essenciais para as novas gerações de redes móveis sem fio.

Uma comunicação robusta pode ser obtida por meio de diversidade temporal e espacial, por meio de codificações espaço-temporais. Com a diversidade espacial, há a transmissão e/ou recepção por múltiplas antenas com espaçamento físico entre elas. A diversidade temporal consiste na transmissão de um mesmo sinal em diferentes intervalos de tempos. Os códigos espaço-temporais unem essas técnicas, proporcionando ganhos significativos ao sistema.

No presente artigo, estuda-se a aplicação de métodos de codificação espaço-temporal do sistema MIMO, baseados em produtos matriciais dos modos de um tensor. À partir da decomposição tensorial PARAFAC, utilizando produtos de Khatri-Rao, em [1-2], é proposta a denominada codificação espaço- temporal de Khatri-Rao (KRST). Por meio da decomposição tensorial de Tucker, tem-se uma codificação tensorial TST [3] e de Kronecker. A performance dessas codificações é analisada em comparação à codificação espaço temporal de Alamouti [4] e ao método V-BLAST [5].

\section{FundamentaÇão teÓRICA}

O modelo PARAFAC [6] (Parallel factors) decompõe um tensor de ordem $N$ em uma soma de $R$ componentes de posto um. O modelo para o tensor de terceira ordem $\mathcal{X} \in \mathbb{C}^{I \times J \times K}$ é dado pela equação:

$$
\mathcal{X} \approx \sum_{r=1}^{R} \mathbf{a}_{r} \circ \mathbf{b}_{r} \circ \mathbf{c}_{r},
$$

Bárbara Oliveira, Universidade Federal do Ceará, Fortaleza-CE, e-mail: barbara@gtel.ufc.br; Michel Gonzaga, Universidade Federal do Ceará, FortalezaCE, email: michel.gonzaga@hotmail.com; Walter Freitas, Universidade Federal do Ceará, Fortaleza-CE, e-mail: walter@gtel.ufc.br. Este trabalho foi financiado pela $\mathrm{CNPq}$. em que o simboliza o produto externo, $r$ é um inteiro positivo, $\mathbf{a}_{\mathbf{r}} \in \mathbb{C}^{I}, \mathbf{b}_{\mathbf{r}} \in \mathbb{C}^{J}$ e $\mathbf{c}_{\mathbf{r}} \in \mathbb{C}^{K}$, para $1 \leq r \leq R$.

Sua representação elemento a elemento é escrita como:

$$
\mathbf{x}_{i j k} \approx \sum_{r=1}^{R} \mathbf{a}_{i r} \circ \mathbf{b}_{j r} \circ \mathbf{c}_{k r}
$$

para $1 \leq i \leq I, 1 \leq j \leq J, 1 \leq k \leq K$.

O tensor também pode ser representado por suas formas matriciais, denominados unfoldings [6], $\mathbf{X}_{(1)}, \mathbf{X}_{(2)}$ e $\mathbf{X}_{(3)}$, em que se utiliza os modos descritos a partir do produto de Khatri-Rao $(\diamond)$, tais que :

$$
\begin{aligned}
& \mathbf{X}_{(1)} \approx \mathbf{A}(\mathbf{C} \diamond \mathbf{B})^{T} \\
& \mathbf{X}_{(2)} \approx \mathbf{B}(\mathbf{C} \diamond \mathbf{A})^{T} \\
& \mathbf{X}_{(3)} \approx \mathbf{C}(\mathbf{B} \diamond \mathbf{A})^{T}
\end{aligned}
$$

O modelo Tucker [6] decompõe um tensor em um produto de um tensor core com matrizes componentes. A representação do modelo para o tensor $\mathcal{X}$ é dada por:

$$
\mathcal{X} \approx \mathcal{G} \times{ }_{1} \mathbf{A} \times{ }_{2} \mathbf{B} \times{ }_{3} \mathbf{C},
$$

em que $\times_{n}$ representa o produto de modo $\mathrm{n}, \mathbf{A} \in \mathbb{C}^{I \times P}, \mathbf{B} \in$ $\mathbb{C}^{J \times Q}$ e $\mathbf{C} \in \mathbb{C}^{K \times R}$ são suas matrizes fatores. $\mathcal{G} \in \mathbb{C}^{P \times Q \times R}$ é seu tensor core, que multiplica-se pelas matrizes fatores ao longo de cada unfolding.

Outra representação possível é:

$$
x_{i j k} \approx \sum_{p=1}^{P} \sum_{q=1}^{Q} \sum_{r=1}^{R} g_{p q r} a_{i p} \circ b_{j q} \circ c_{k r},
$$

para $1 \leq i \leq I, 1 \leq j \leq J, 1 \leq k \leq K$.

$\mathrm{O}$ modelo pode ser representado por meio de seus ulfoldings [6] $\mathbf{X}_{(1)}, \mathbf{X}_{(2)}$ e $\mathbf{X}_{(3)}$, com produtos de Kronecker $(\otimes)$ :

$$
\begin{aligned}
& \mathbf{X}_{(1)} \approx \mathbf{A G}_{P \times R Q}(\mathbf{C} \otimes \mathbf{B})^{T} \\
& \mathbf{X}_{(2)} \approx \mathbf{B G}_{Q \times R P}(\mathbf{C} \otimes \mathbf{A})^{T} \\
& \mathbf{X}_{(3)} \approx \mathbf{C G}_{R \times Q P}(\mathbf{B} \otimes \mathbf{A})^{T}
\end{aligned}
$$

\section{Metodologia}

\section{A. Modelo do sistema}

O sistema MIMO é composto por $M_{t}$ antenas transmissoras e $M_{r}$ antenas receptoras. Tem-se como matriz de símbolo $\mathbf{S} \in \mathbb{C}^{D \times M_{t}}$, com $D$ símbolos multiplexados em $M_{t}$ antenas, assumindo um canal com desvanecimento Rayleigh $\mathbf{H} \in \mathbb{C}^{M r \times M t}$. Os esquemas de codificação propostos para o sistema são descritos a seguir: 
1) Codificação espaço-temporal de Khatri-Rao: O modelo é baseado na proposta de codificação KRST em [1][2]. A matriz de símbolo $\mathbf{S}$ é codificada pela matriz de código $\mathbf{C}$ $\in \mathbb{C}^{K \times M_{t}}$. Por meio do produto de Khatri-Rao, espalha-se o sinal transmitido por $K$ blocos de tempo.

A matriz unfolding modo 3 do sinal recebido, $\mathbf{Y} \in$ $\mathbb{C}^{K \times D M_{r}}$, definida de acordo com a equação (5), é dada por:

$$
\mathbf{Y}_{K \times D M_{r}}=\mathbf{C}(\mathbf{S} \diamond \mathbf{H})^{T}+\mathbf{N}_{K \times D M_{r}},
$$

em que $\mathbf{N} \in \mathbb{C}^{K \times D M_{r}}$ é a matriz de ruído.

Assumindo a matriz de codificação conhecida, na decodificação, estima-se a matriz de canal $\mathbf{H}$ e a matriz de símbolo $\mathbf{S}$. Primeiro, toma-se a inversa da equação (11) e, assumindo que C é uma matriz de colunas ortonormais, de forma que tem-se $\mathbf{C C}^{H}=\mathbf{I}$, em que $\mathbf{C}^{H}$ indica a Hermitiana de $\mathbf{C}$, obtemos:

$$
\mathbf{C}^{H} \mathbf{Y}_{P \times N M}=(\widehat{\mathbf{S} \diamond \mathbf{H}})^{T},
$$

omitindo-se a matriz de ruído para simplificação da notação. Realiza-se a fatoração do produto de Khatri-Rao em Least Squares (LS), fatorando o tensor em sua solução fechada. Então, estima-se as matrizes $\mathbf{S}$ e $\mathbf{H}$ por meio do algoritmo de decomposição em valores singulares (SVD) de fatoração de Khatri-Rao [2].

2) Codificação espaço-temporal de Kronecker: O modelo é baseado na codificação tensorial espaço-temporal (TST) [3]. A matriz de símbolo $S$ é codificada pelo tensor $\mathcal{G} \in$ $\mathbb{C}^{M_{r} \times M_{t} \times Q}$, o código TST, e pela matriz $\mathbf{C} \in \mathbb{C}^{P \times Q}$, o código KronST, ambos gerados à partir de DFT. As dimensões $P$ e $Q$ proporcionam espalhamento no tempo e espaço para a matriz de símbolo $\mathbf{S}$.

Tomando o terceiro modo do tensor do sinal recebido $\mathbf{Y}_{P \times D M_{r}}$, definido à partir de (10):

$$
\mathbf{Y}_{P \times D M_{r}}=\mathbf{C G}_{Q \times M_{r}^{2}}(\mathbf{S} \otimes \mathbf{H})^{T}+\mathbf{N}_{P \times D M_{r}} .
$$

Tomando a transposta da equação (13), fatora-se o produto Kronecker $(\mathbf{S} \otimes \mathbf{H})^{T}$ por meio do algoritmo LS:

$$
(\mathbf{G C})^{\dagger} \mathbf{Y}_{P \times D M_{r}}=(\widehat{\mathbf{S} \otimes \mathbf{H}})^{T} \text {. }
$$

Então, de forma semelhante ao modelo com o código KRST, utiliza-se o algoritmo SVD de fatoração de Kronecker [2] para estimar as matrizes $\mathbf{S}$ e $\mathbf{H}$.

\section{RESUltados}

O sistema MIMO foi implementado para um cenário de duas antenas transmissoras e duas antenas receptoras com codificação KRST, KronST, em seu modelo V-BLAST e com codificação Alamouti. Utilizou-se a modulação 4QAM e ruído aditivo Gaussiano branco com variância $\sigma^{2}$. Foi calculada a relação sinal-ruído $\left(E_{s} / N_{0}\right)$ em termos da taxa de erro de símbolo (SER) e erro quadrático médio (NMSE) da estimação do canal dos algoritmos KRST e KronST.

Na Figura 1, pode-se ver que os menores valores de SER correspondem ao algoritmo KronST. Para o KRST, temos valores iniciais mais altos, mas que convergem rapidamente em relação aos modelos clássicos. Na Figura 2, temos que o algoritmo KronST possui melhor estimação do canal que o KRST.

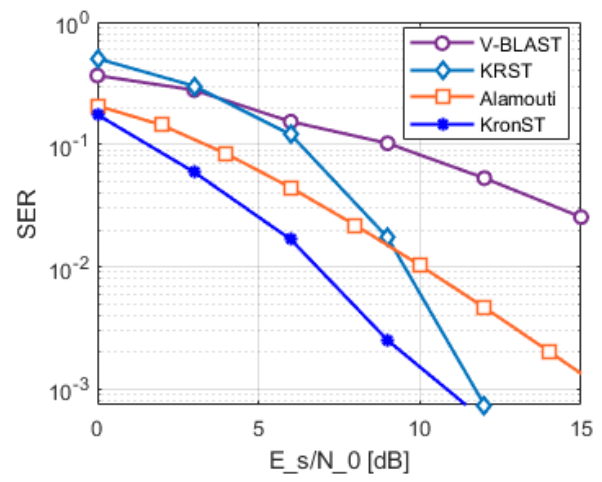

Fig. 1. Performance dos modelos para modulação 4QAM.

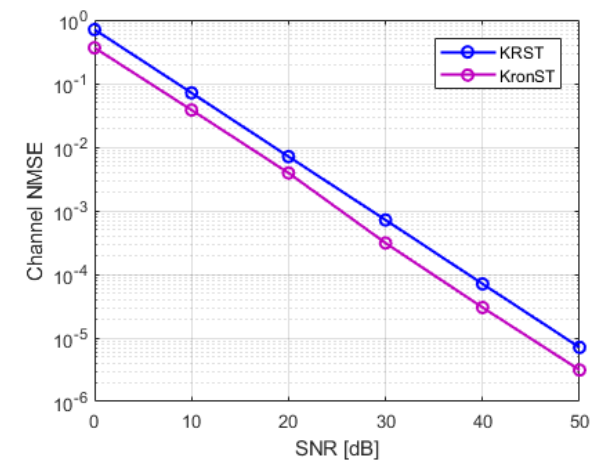

Fig. 2. NMSE da estimação de canal pelo algoritmo KRST e KronST.

\section{CONCLUSÃO}

Os resultados obtidos mostram que a utilização de codificações espaço-temporais baseadas em produtos tensoriais aumentam a performance do sistema MIMO em relação a outros algoritmos clássicos. Esse resultado se destaca ainda mais pelo fato de que, na simulação, os algoritmos Alamouti e V-BLAST assumiram canal já conhecido e, os algoritmos tensoriais com estimação de canal apresentaram resultados próximos ou superiores. Dessa forma, o trabalho apresentado confirma a eficácia da aplicação de modelos tensoriais para telecomunicações.

\section{REFERÊNCIAS}

[1] N. D. Sidiropoulos and R. S. Budampati, "Khatri-Rao space-time codes,"in IEEE Transactions on Signal Processing, vol. 50,no.10, Oct.2002.

[2] W. Jr, G. Favier, A. de Almeida. Generalized Khatri-Rao and Kronecker Space-Time Coding for MIMO Relay Systems with Closed-Form SemiBlind Receivers. Signal Processing, vol. 151, pp. 19-31, 2018.

[3] G. Favier, M. N. da Costa, A. de Almeida, J. M. Romano. Tensor space-time (TST) coding for MIMO wireless communication systems, Signal Processing, Volume 92, Issue 4, 2012.

[4] S. M. Alamouti, "A simple transmit diversity technique for wireless communications,", IEEE Journal on Selected Areas in Communications, vol. 16 , no. 8, pp. 1451-1458, 1998.

[5] V. Bhojak and A. Sharma, "MIMO Wireless Systems: V-BLAST Architecture,"2013 Third International Conference on Advanced Computing and Communication Technologies (ACCT), pp.215-220, 2013.

[6] T. G. Kolda and B. W. Bader, "Tensor decompositions and applications," SIAM review, vol. 51, no. 3, 2009.

[7] A. Smilde, R. Bro, and P. Geladi, Multi-way Analysis: Applications in the Chemical Sciences. New York: John Wiley \& Sons Ltd, 2004. 